\title{
Effects of All-Atom Molecular Mechanics Force Fields on Amyloid Peptide Assembly: The Case of PHF6 Peptide of Tau Protein
} (SUPPORTING INFORMATION)

\author{
Viet Hoang Man, ${ }^{1}$ Xibing He, ${ }^{1}$ Jie Gao, ${ }^{2}$ and Junmei Wang ${ }^{1 *}$ \\ ${ }^{1}$ Department of Pharmaceutical Sciences and Computational Chemical Genomics Screening \\ Center, School of Pharmacy, University of Pittsburgh, Pittsburgh, PA 15261, USA. \\ 2 Department of Neuroscience, The Ohio State University Wexner Medical Center, Columbus, \\ $\mathrm{OH} 43210$, USA. \\ E-mail: junmei.wang@pitt.edu
}

\section{Replica exchange simulation}

For each 2PHF6 system, the replica exchange simulation (REMD) was performed using 16 replicas, which covers a temperature range from $305 \mathrm{~K}$ to $383 \mathrm{~K}$. The initial structures of REMD were the initial structures of the first 16 of $100 \mathrm{MD}$ trajectories. The temperatures of replicas were determined by using the method proposed by Patriksson and van der Spoel (Phys. Chem. Chem. Phys., 2008, 10, 2073-2077). The requested acceptance ratio was around $20 \%$. Exchanges between replicas were attempted every 2 ps, large enough compared to the coupling time of the heat bath. Each replica was run for $200 \mathrm{~ns}$, and the data were collected every 10 ps. The first $20 \mathrm{~ns}$ of all trajectories were excluded for analysis. All other simulation parameters were set the same as those ones of the conventional MD simulation (see the main tex). 

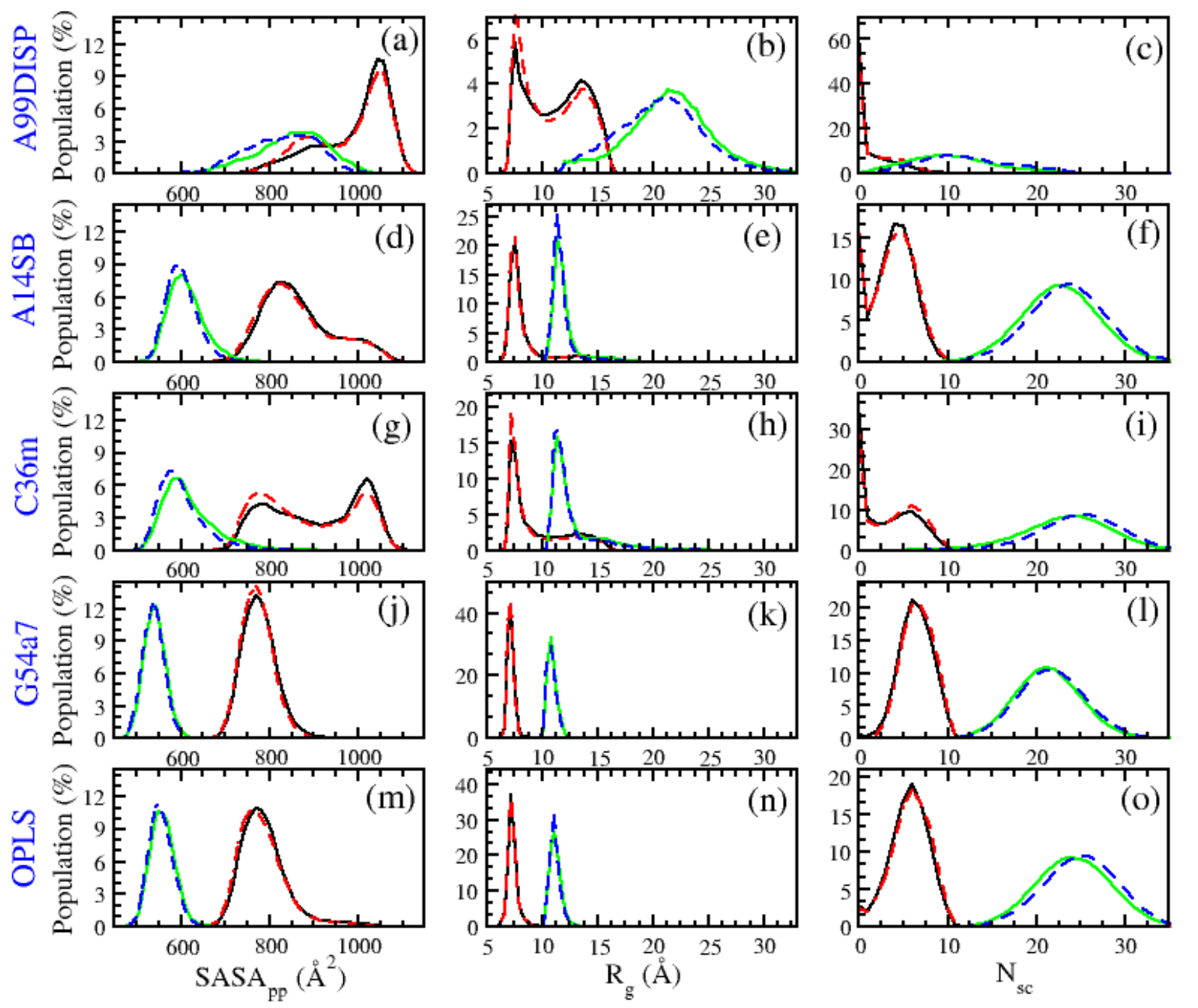

Figure S1: The distribution of surface accessible solvent area per peptide ( $\mathrm{SASA} \mathrm{Ap}_{\mathrm{p}}$ ), radii of gyration $\left(\mathrm{R}_{g}\right)$ and number of intermolecular side chain-side chain contact $\left(N_{s c}\right)$ of 2PHF6 and 8PHF6 in corresponded systems. The data of 2PHF6 systems were calculated from the simulation periods, from $50 \mathrm{~ns}$ to $125 \mathrm{~ns}$ (solid black lines) and from $125 \mathrm{~ns}$ to $200 \mathrm{~ns}$ (dash red lines), of 100 trajectories. The data of 8PHF6 systems were calculated from the simulation periods, from 200 ns to 600 ns (solid green lines) and from $600 \mathrm{~ns}$ to $1000 \mathrm{~ns}$ (dash blue lines), of 20 trajectories. 

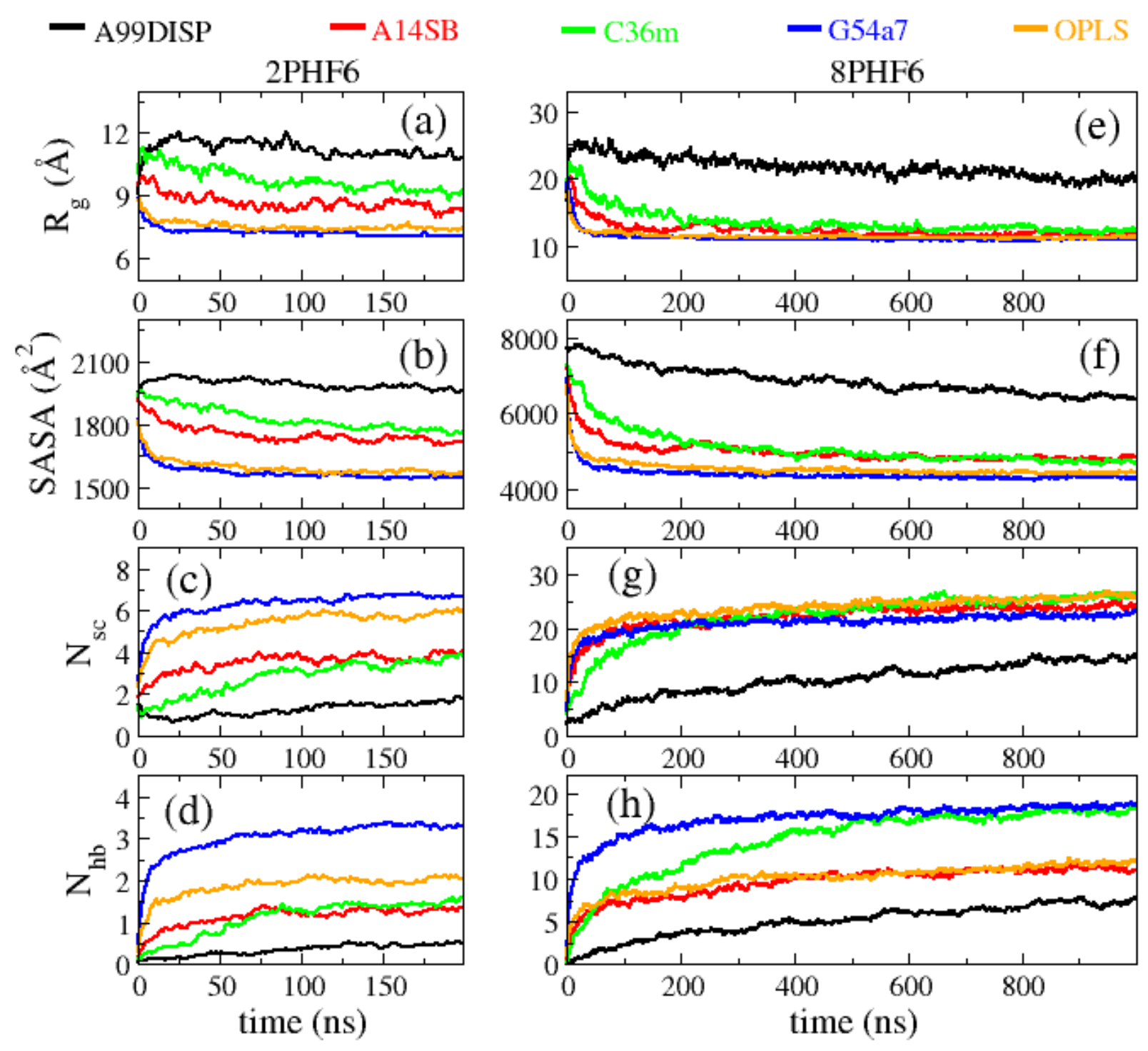

Figure S2: Evolution of the numbers of intermolecular side chain-side chain contacts $\left(\mathrm{N}_{\mathrm{sc}}\right)$, the numbers of intermolecular of main chain hydrogen bonds $\left(\mathrm{N}_{\mathrm{hb}}\right)$, surface accessible solvent area (SASA) and radii of gyration $\left(\mathrm{R}_{\mathrm{g}}\right)$ of 2 PHF6 and 8PHF6 along the MD simulation time using different force fields. 

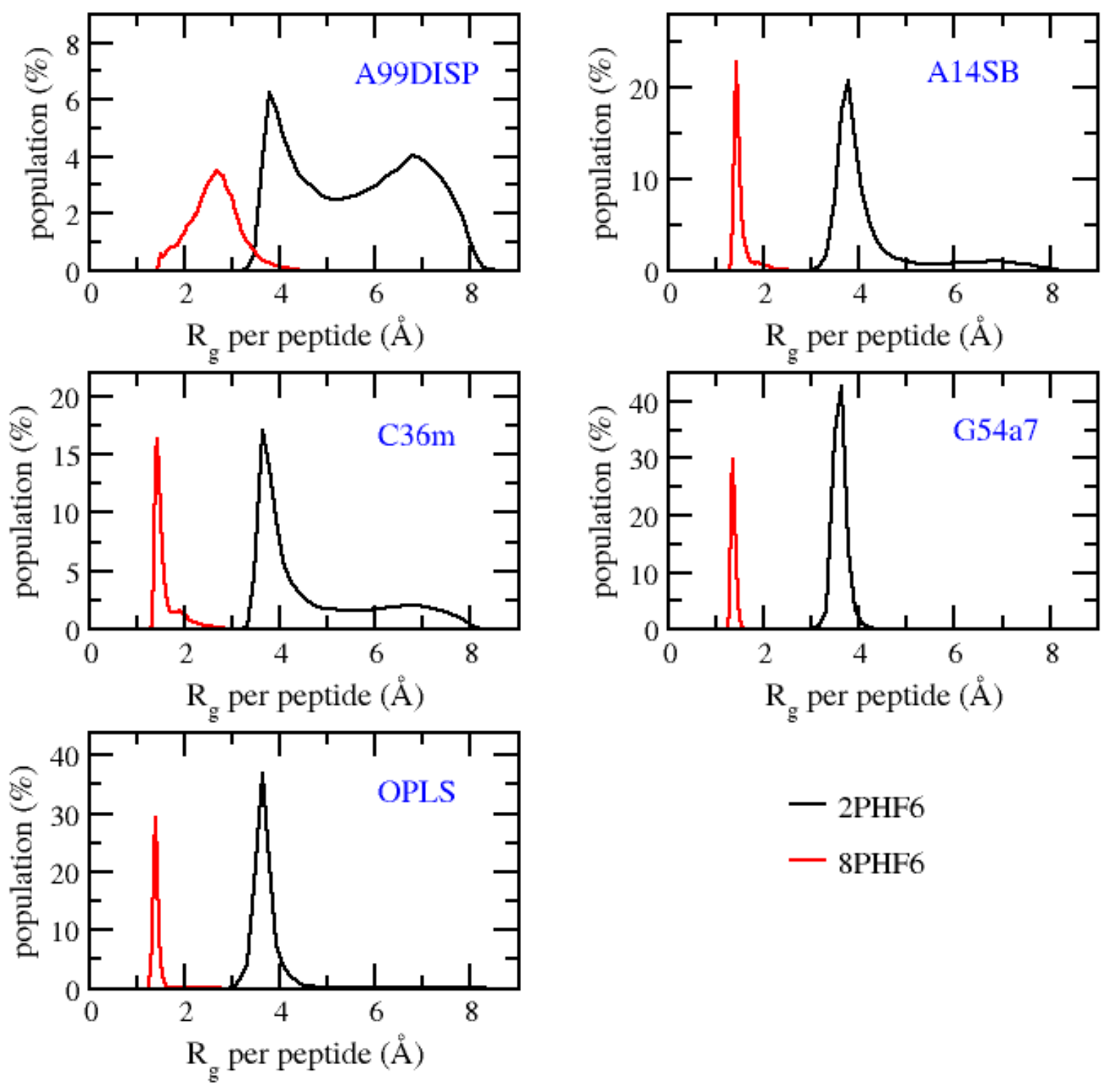

- 2PHF6

- 8PHF6

Figure S3: The distribution of gyration radius per peptide of 2PHF6 and 8PHF6 in corresponded systems. 


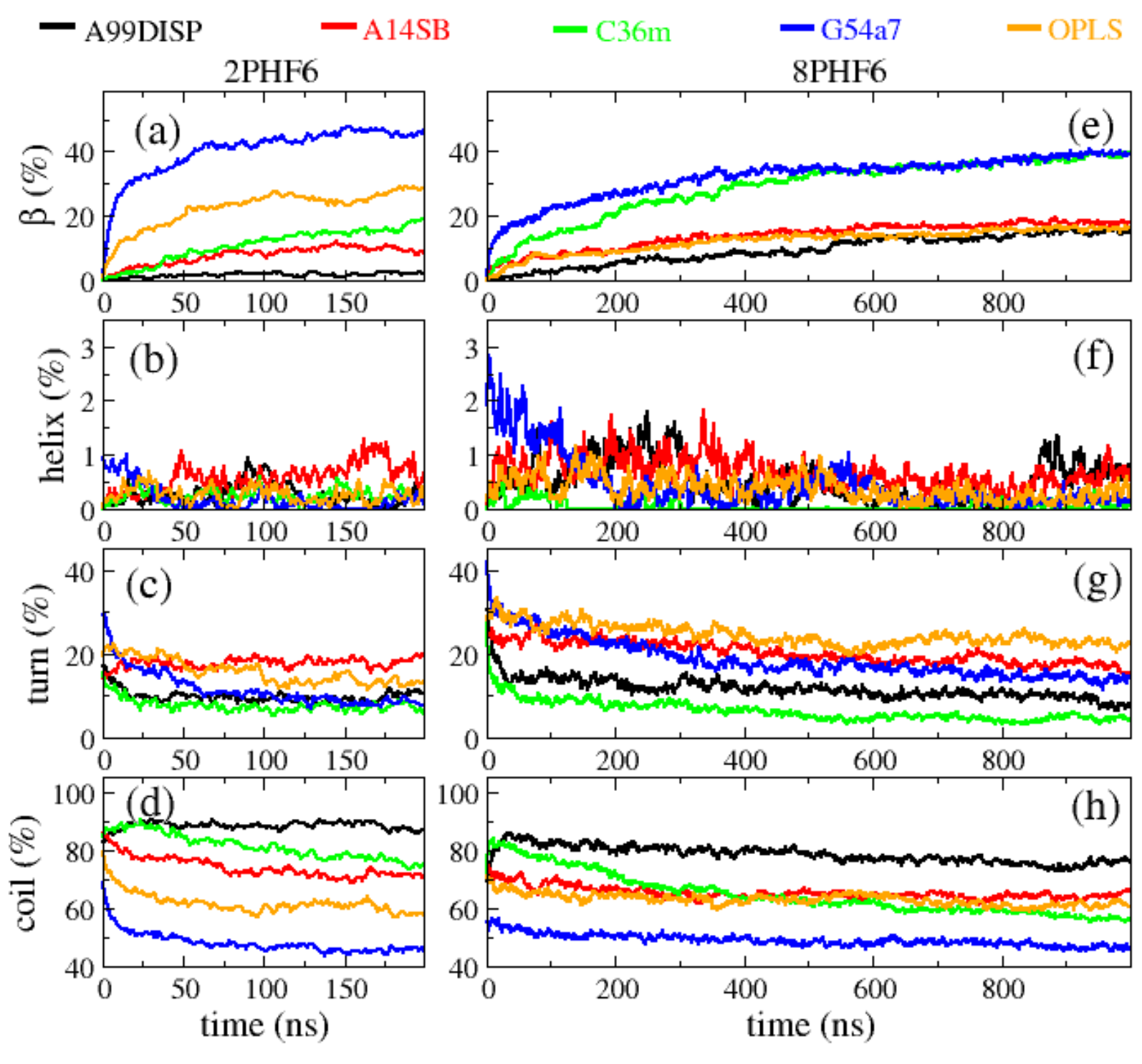

Figure S4: Evolution of the secondary structures of PHF6 peptides in different systems along the MD simulation time using different force fields 


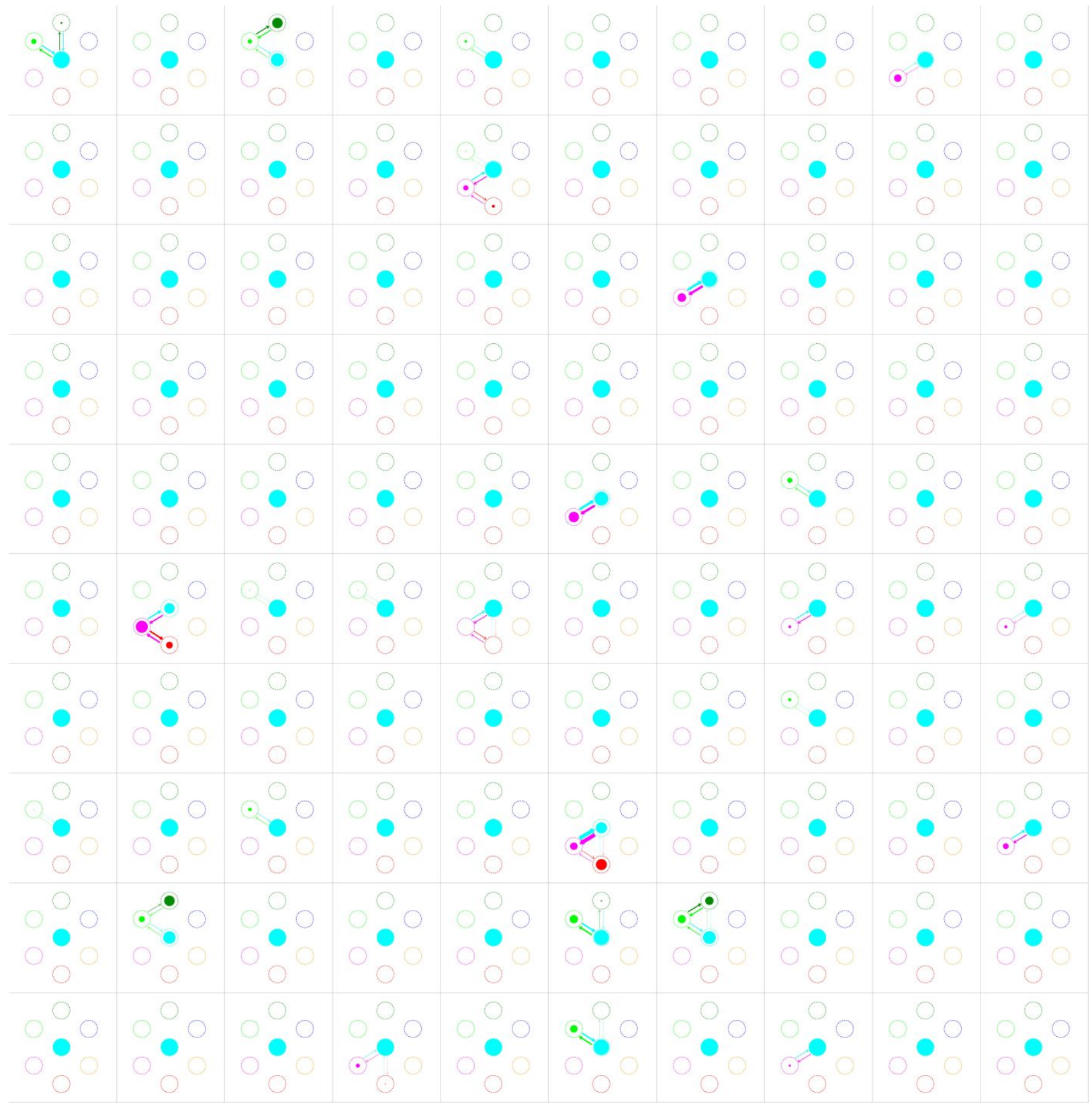

Figure S5: The populations of the seven states and the state transitions of the PHF6 dimer in the 2PHF6 system with A99SB-DISP force field. The analyses were performed for $100 \mathrm{MD}$ trajectories and each was shown in a rectangle block. The definitions and representations of the seven states are presented in Figure 1. 


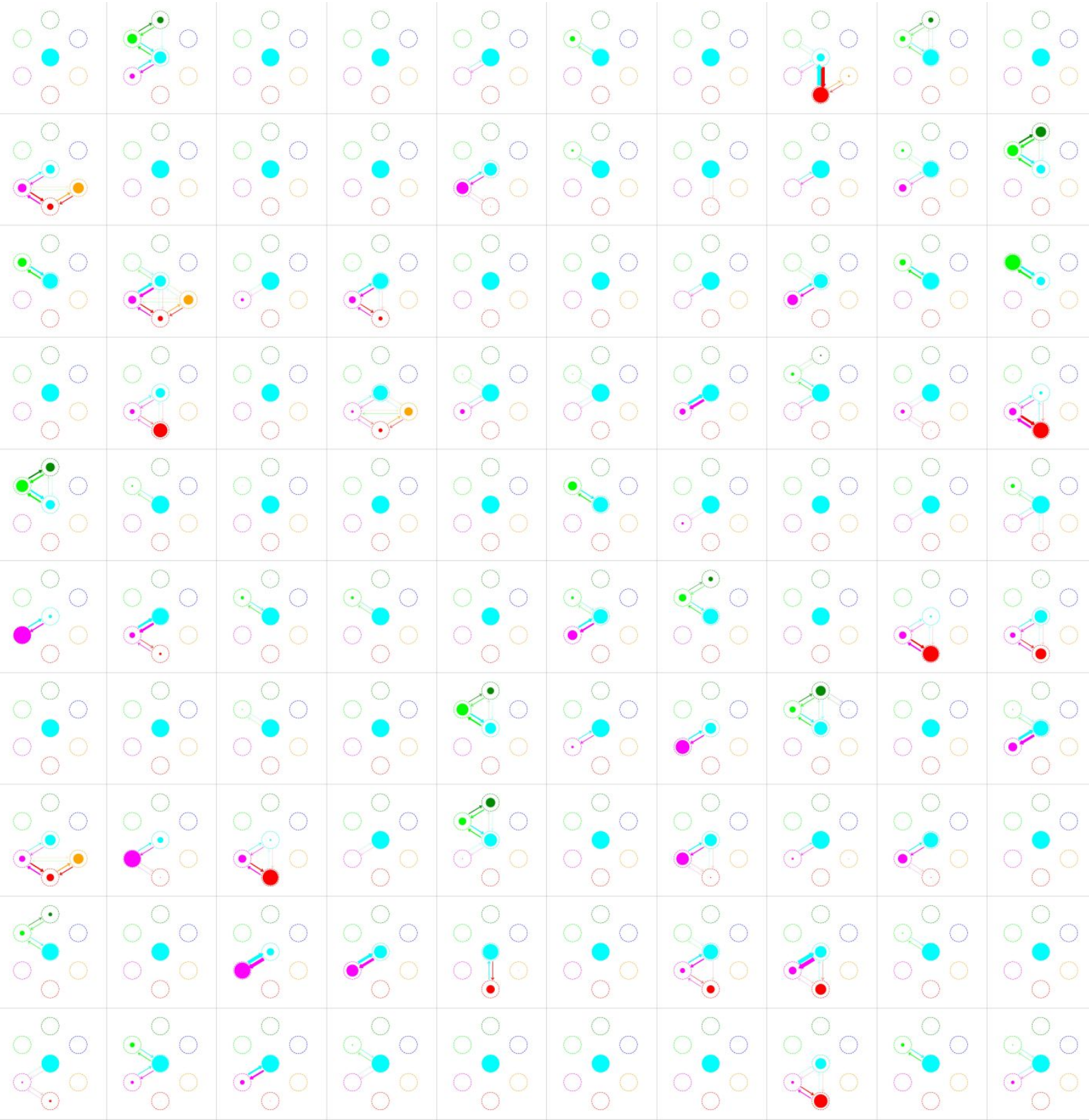

Figure S6: The populations of the seven states and the state transitions of the PHF6 dimer in the 2PHF6 system with A14SB force field. The analyses were performed for $100 \mathrm{MD}$ trajectories and each was shown in a rectangle block. The definitions and representations of the seven states are presented in Figure 1. 


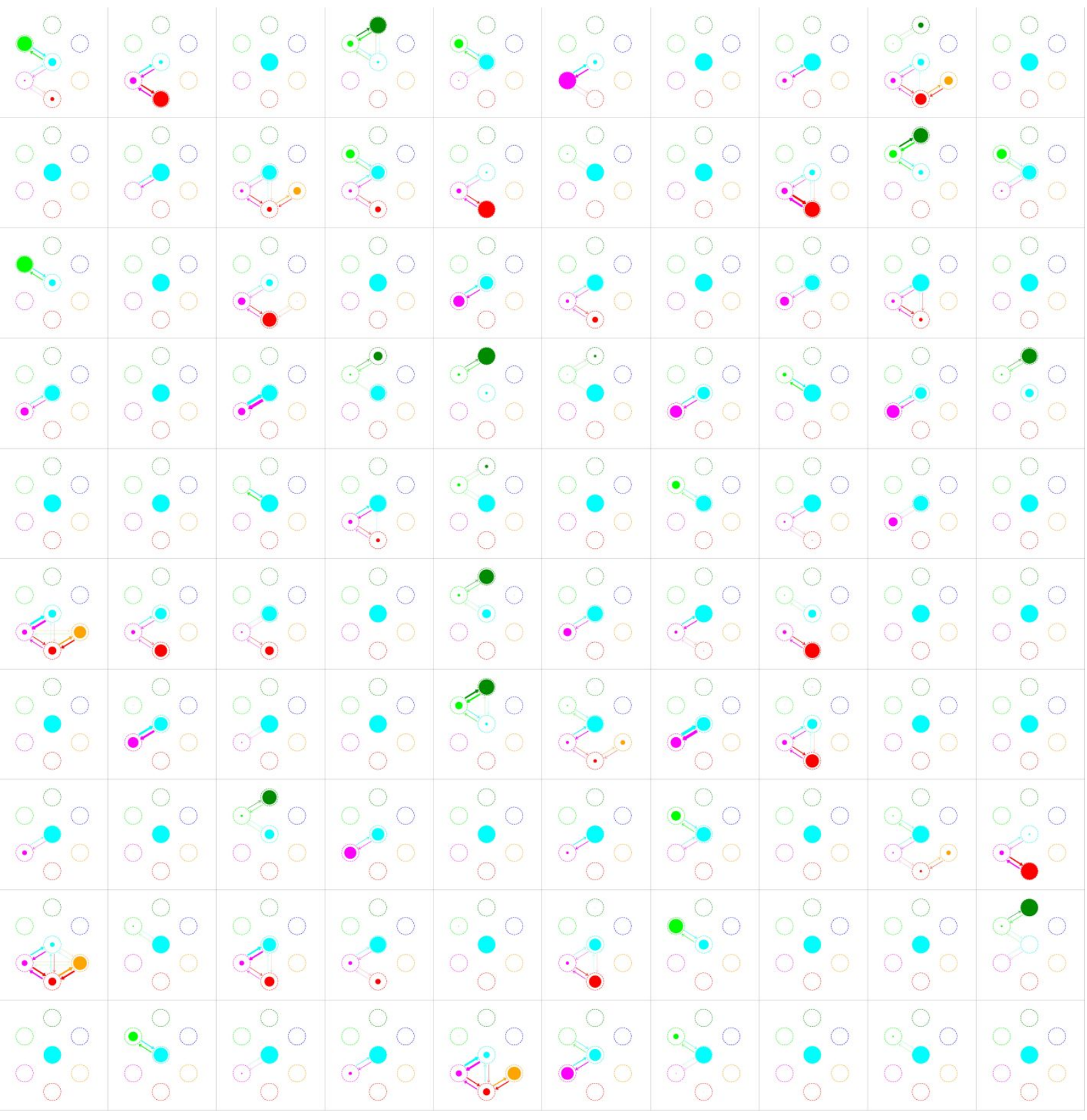

Figure S7: The populations of the seven states and the state transitions of the PHF6 dimer in the 2PHF6 system with $\mathrm{C} 36 \mathrm{~m}$ force field. The analyses were performed for $100 \mathrm{MD}$ trajectories and each was shown in a rectangle block. The definitions and representations of the seven states are presented in Figure 1. 


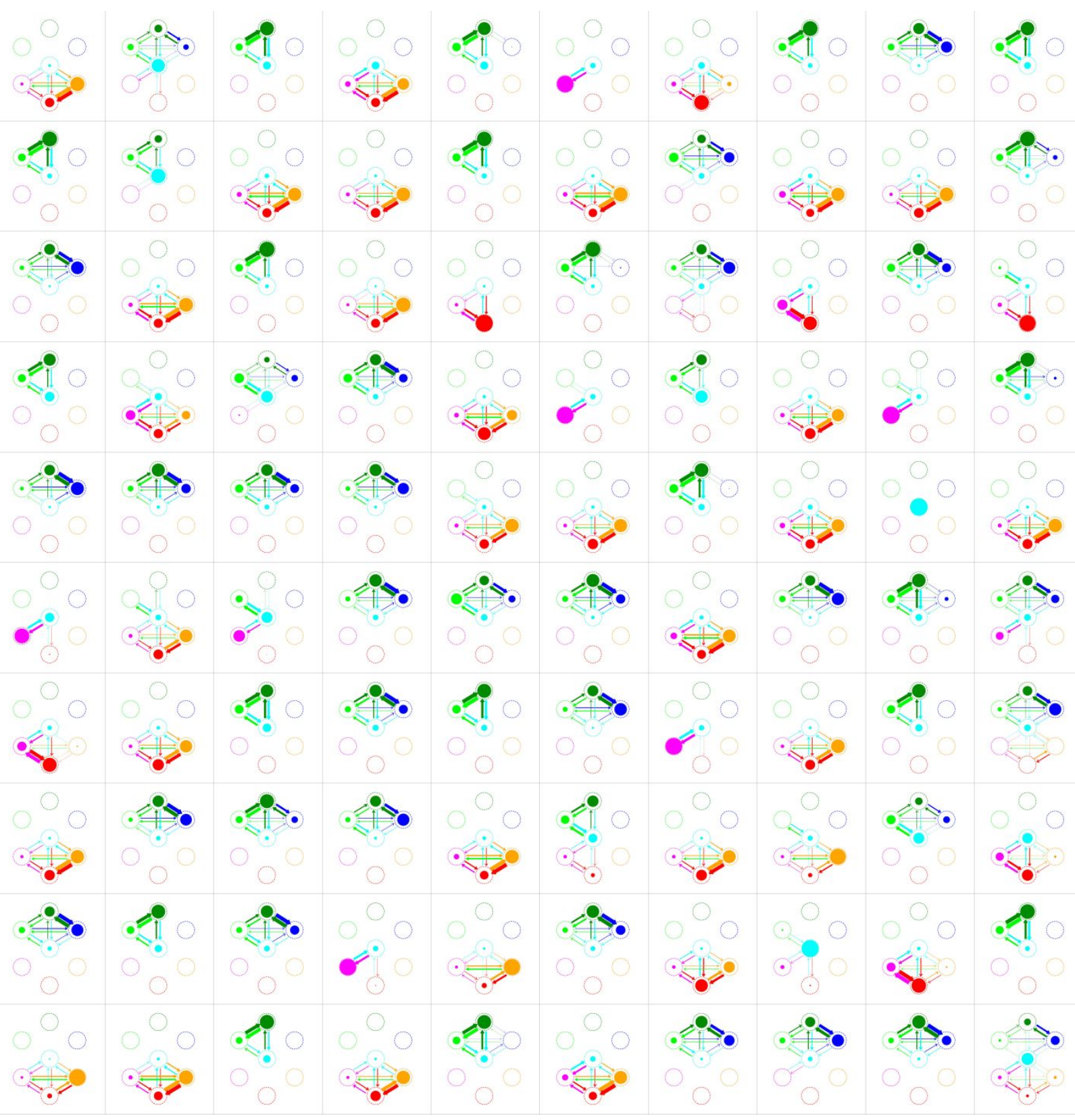

Figure S8: The populations of the seven states and the state transitions of the PHF6 dimer in the 2PHF6 system with G54a7 force field. The analyses were performed for $100 \mathrm{MD}$ trajectories and each was shown in a rectangle block. The definitions and representations of the seven states are presented in Figure 1. 


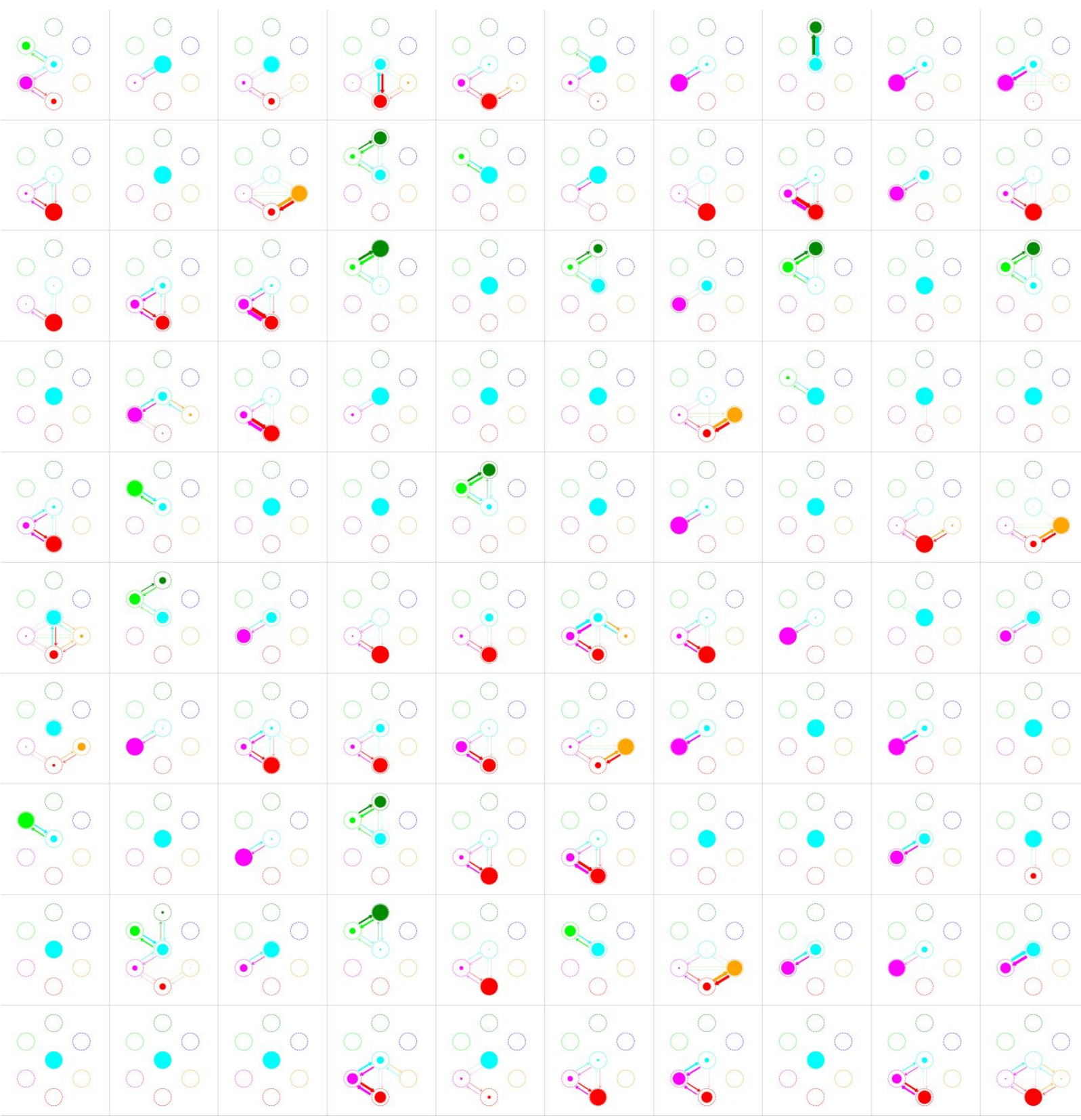

Figure S9: The populations of the seven states and the state transitions of the PHF6 dimer in the 2PHF6 system with OPLS force field. The analyses were performed for $100 \mathrm{MD}$ trajectories and each was shown in a rectangle block. The definitions and representations of the seven states are presented in Figure 1. 


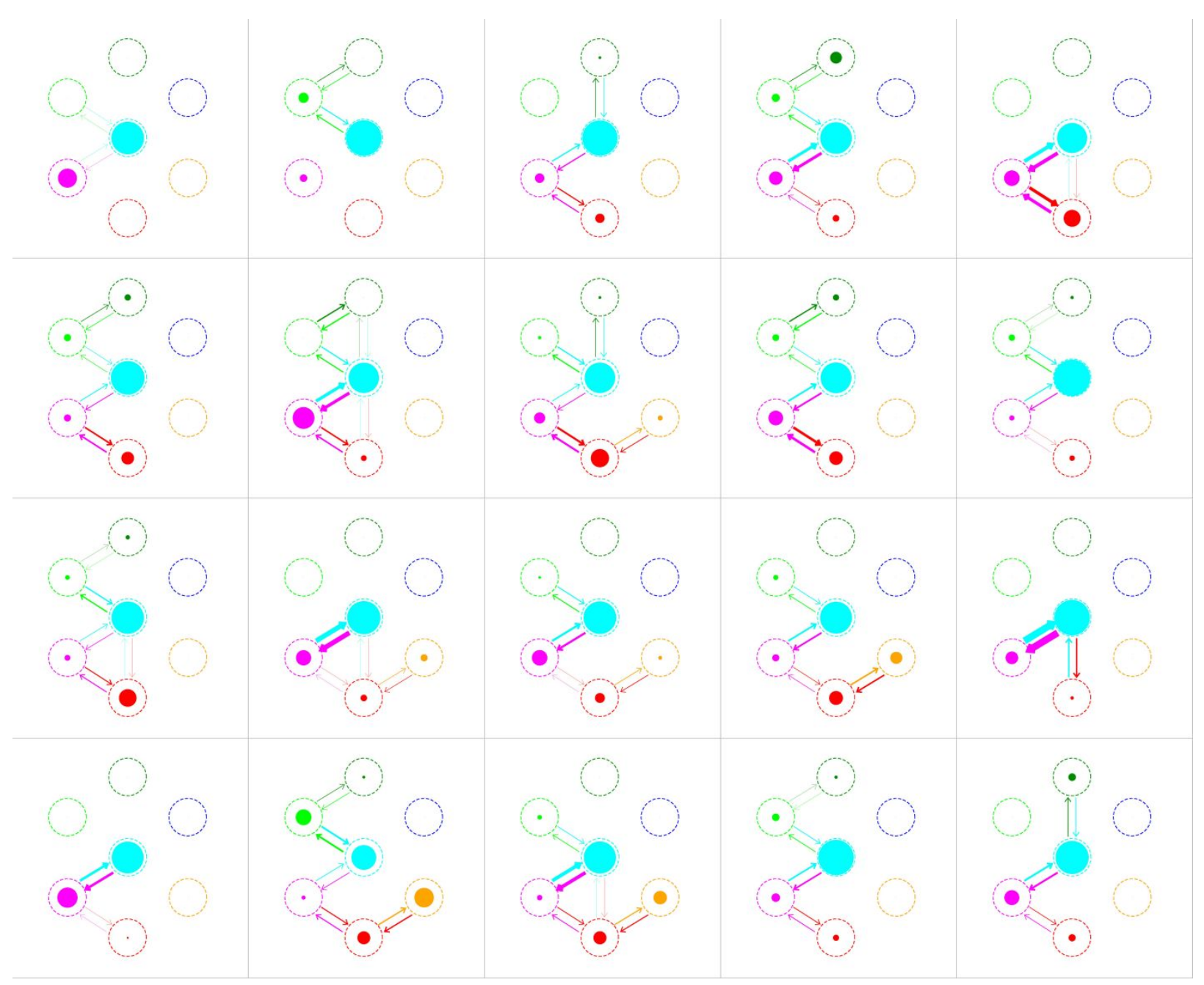

Figure S10: The populations of the seven states and the state transitions of the PHF6 dimer in the 8PHF6 system with A99SB-DISP force field. The analyses were performed for $20 \mathrm{MD}$ trajectories and each was shown in a rectangle block. The definitions and representations of the seven states are presented in Figure 1. 


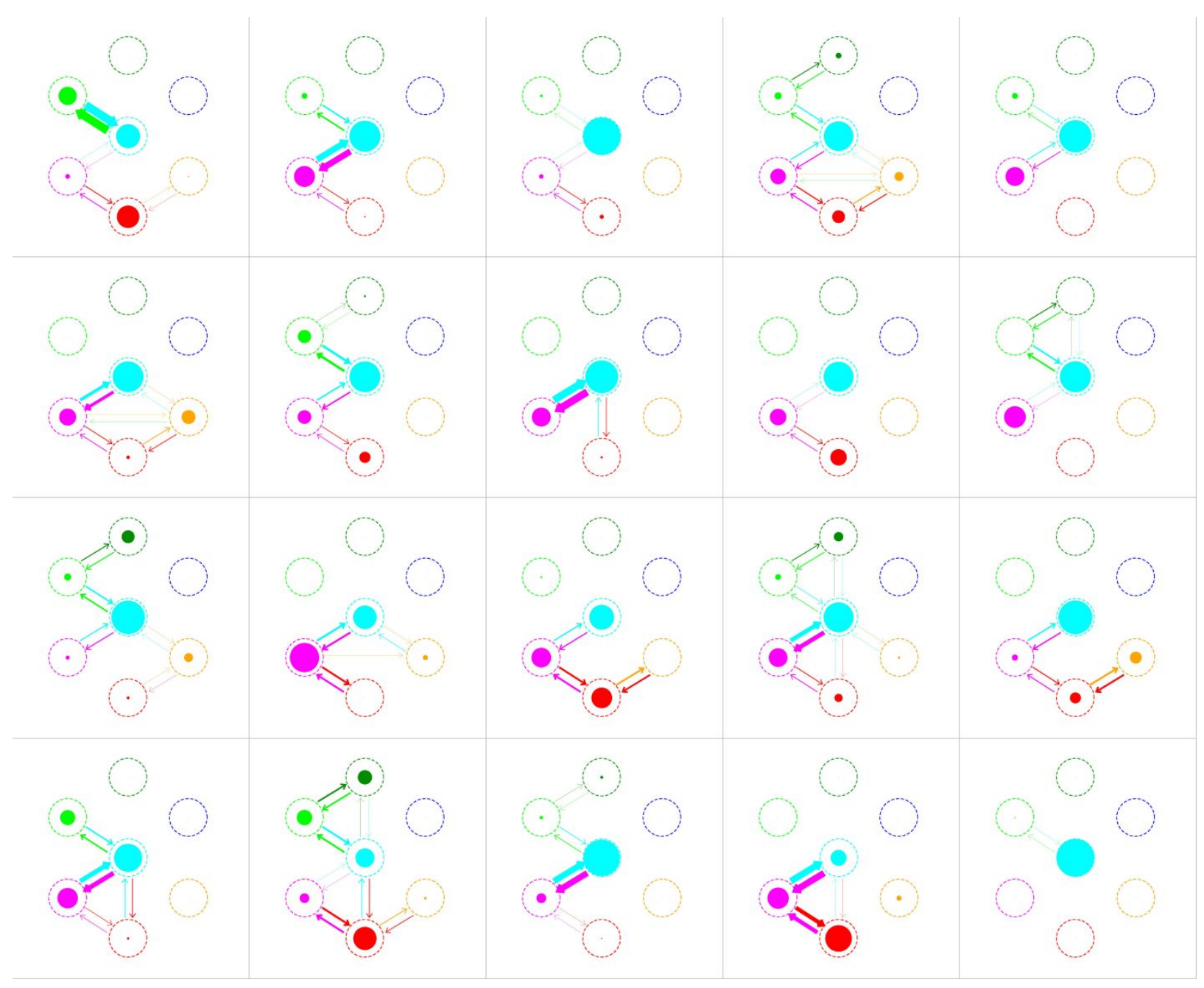

Figure S11: The populations of the seven states and the state transitions of the PHF6 dimer in the 8PHF6 system with A14SB force field. The analyses were performed for $20 \mathrm{MD}$ trajectories and each was shown in a rectangle block. The definitions and representations of the seven states are presented in Figure 1. 


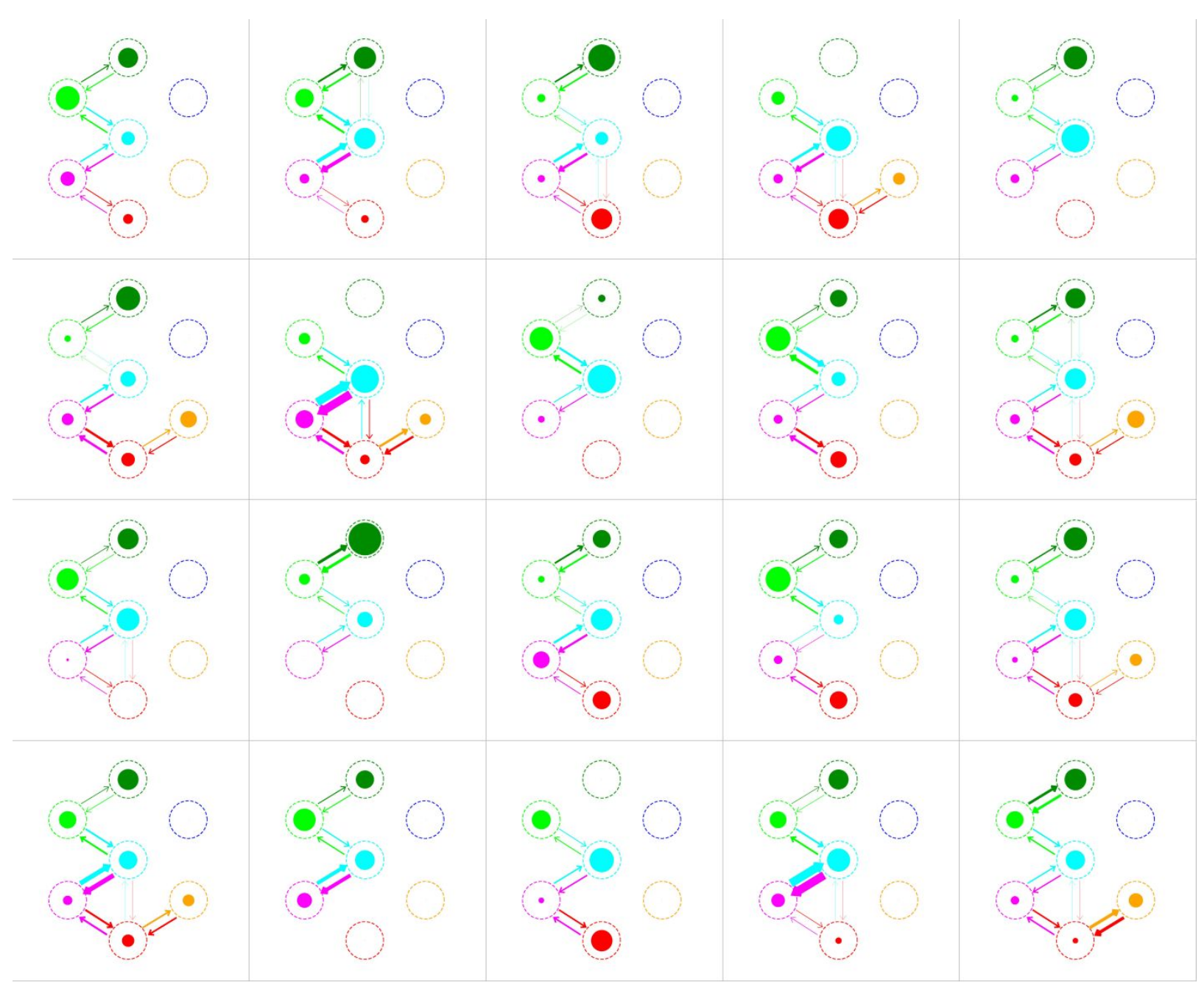

Figure S12: The populations of the seven states and the state transitions of the PHF6 dimer in the 8PHF6 system with C36m force field. The analyses were performed for $20 \mathrm{MD}$ trajectories and each was shown in a rectangle block. The definitions and representations of the seven states are presented in Figure 1. 

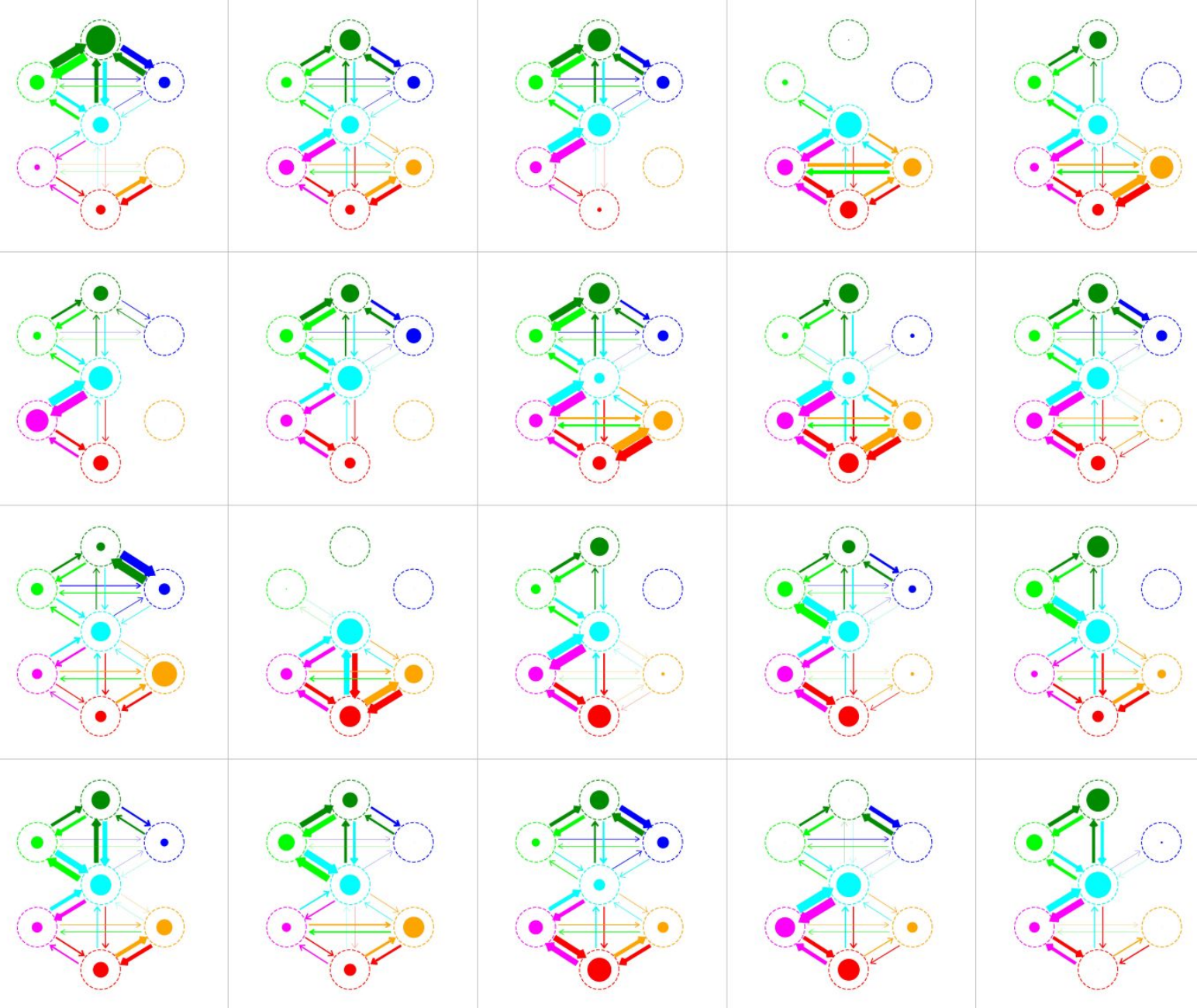

Figure S13: The populations of the seven states and the state transitions of the PHF6 dimer in the 8PHF6 system with G54a7 force field. The analyses were performed for $20 \mathrm{MD}$ trajectories and each was shown in a rectangle block. The definitions and representations of the seven states are presented in Figure 1. 


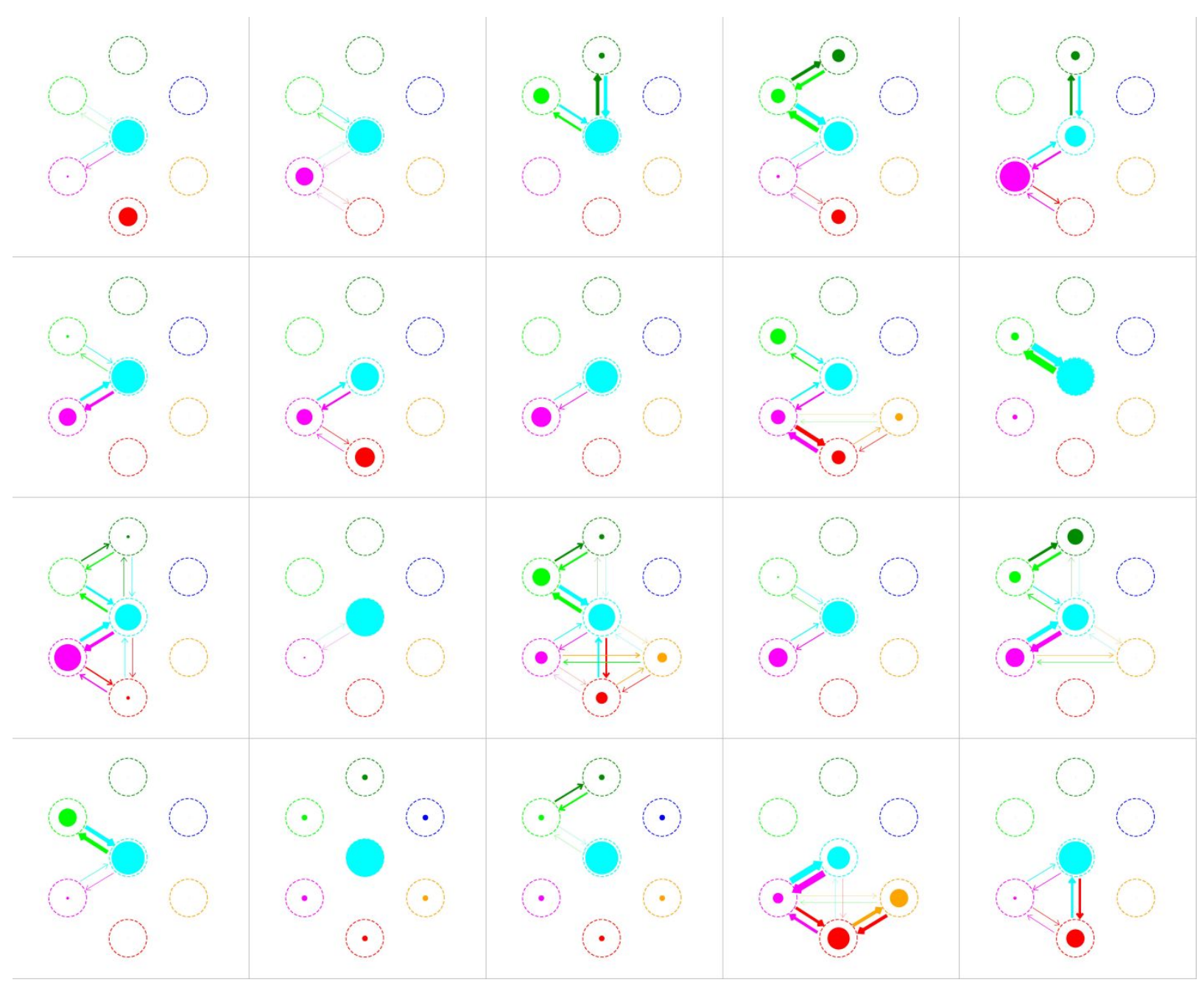

Figure S14: The populations of the seven states and the state transitions of the PHF6 dimer in the 8PHF6 system with OPLS force field. The analyses were performed for $20 \mathrm{MD}$ trajectories and each was shown in a rectangle block. The definitions and representations of the seven states are presented in Figure 1. 


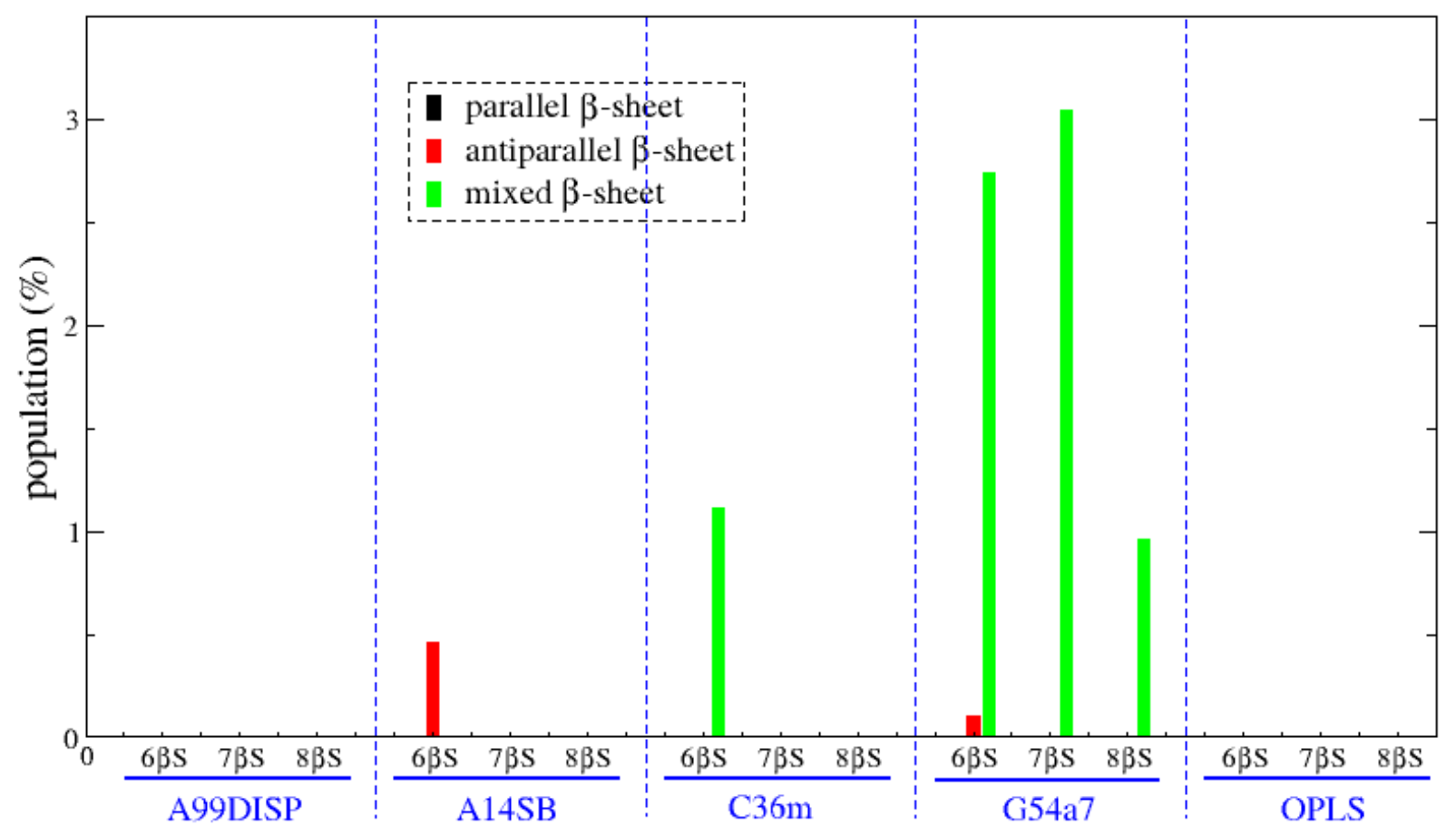

Figure S15: The populations of parallel, antiparallel and mixed type of $\beta$-sheet structures with different number of strands found sizes formed in the 8PHF6 system in using different force fields. A $\beta$-sheet structure containing i peptides is denoted as i $\beta$ S. For the 8 PHF6 system that has having 8 monomeric peptides, $i$ takes values of 2 to 8 . This figure shows the distributions of three types for $i \beta S(6 \leq i \leq 8)$. 

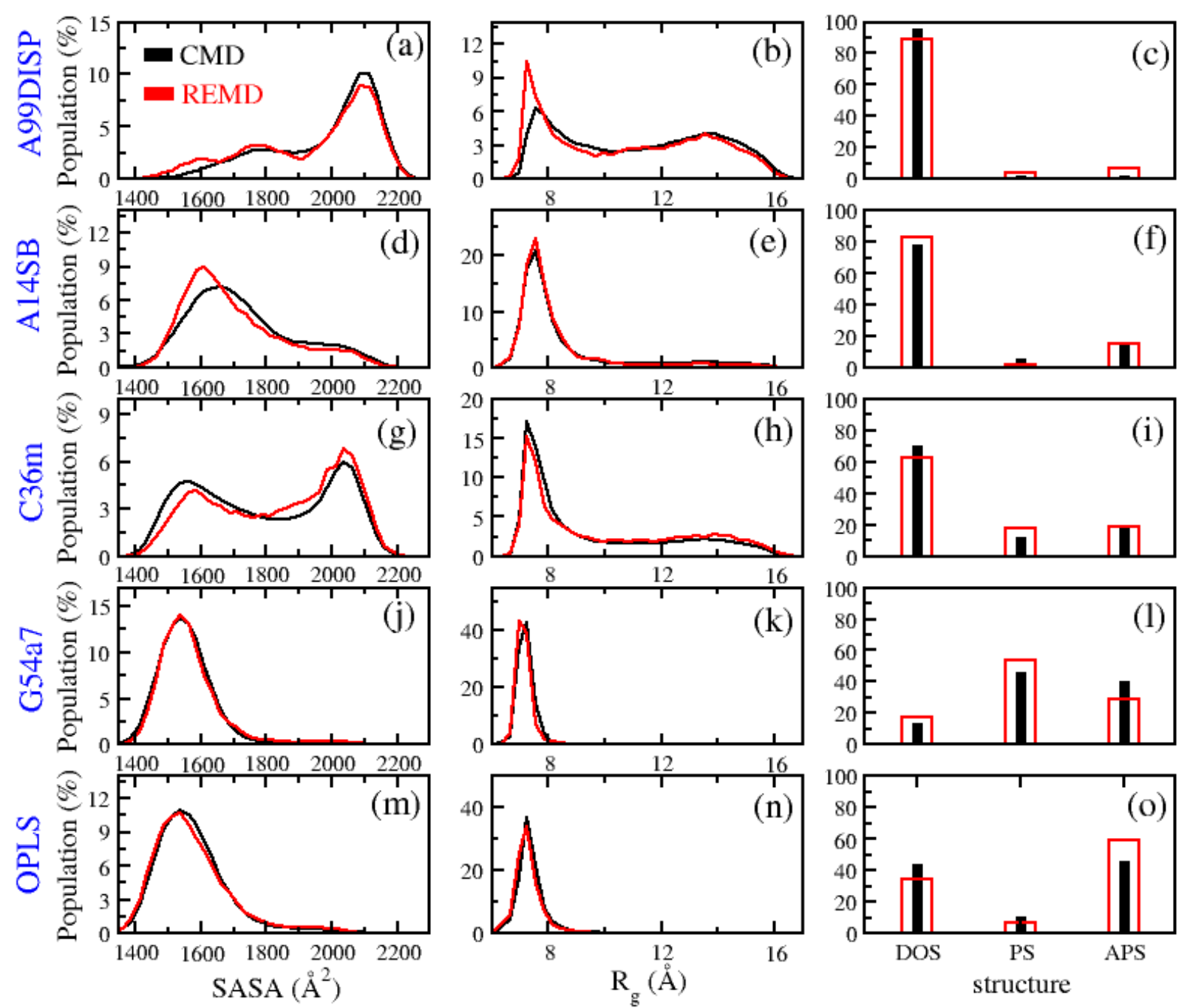

Figure S16: The comparison between conventional MD (CMD) simulation with 100 trajectories (black) and REMD simulation (red) for 2PHF6 systems. The data is shown for the population of SASA, gyration radius, and DOS, PS and APS structures. The REMD conformation data were collected when the replica temperature is $310 \mathrm{~K}$, at which CMD were performed. The agreement between CMD and REMD confirms the convergence of conformational sampling of the multirun CMD simulation. 